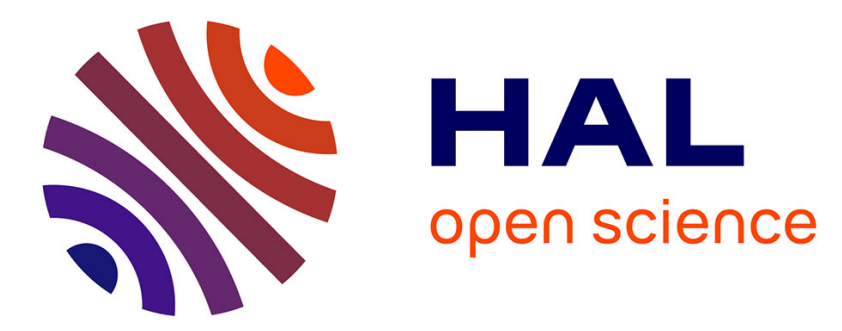

\title{
Un type d'implantation industrielle en milieu rural : les mines de Montredon-Labessonnié (Tarn) \\ Georges Bertrand
}

\section{To cite this version:}

Georges Bertrand. Un type d'implantation industrielle en milieu rural: les mines de MontredonLabessonnié (Tarn). Revue Géographique des Pyrénées et du Sud-Ouest, 1959, 30 (2), pp.169-178. 10.3406/rgpso.1959.1542. hal-02570344

\section{HAL Id: hal-02570344 \\ https://hal.science/hal-02570344}

Submitted on 12 May 2020

HAL is a multi-disciplinary open access archive for the deposit and dissemination of scientific research documents, whether they are published or not. The documents may come from teaching and research institutions in France or abroad, or from public or private research centers.
L'archive ouverte pluridisciplinaire HAL, est destinée au dépôt et à la diffusion de documents scientifiques de niveau recherche, publiés ou non, émanant des établissements d'enseignement et de recherche français ou étrangers, des laboratoires publics ou privés. 


\section{Un type d'implantation industrielle en milieu rural : les mines de Montredon-Labessonnié (Tarn)}

\section{Georges Bertrand}

\section{Citer ce document / Cite this document :}

Bertrand Georges. Un type d'implantation industrielle en milieu rural : les mines de Montredon-Labessonnié (Tarn). In: Revue géographique des Pyrénées et du Sud-Ouest, tome 30, fascicule 2, 1959. pp. 169-178;

doi : https://doi.org/10.3406/rgpso.1959.1542

https://www.persee.fr/doc/rgpso_0035-3221_1959_num_30_2_1542

Fichier pdf généré le 05/04/2018 
UN TYPE D'IMPLANTATION INDUSTHILLLE RN MIIILC RUHAL:

\section{LES MINES DE MONTREDON-LABESSONNIE (TARN) par Genrges BERTIAND}

Transition entre les hautes terres cristallines du Lacaunais el les collines molassiques du bassin de Castres, le Montredonnais se présenie comme un monotone plateau de schistes primaires (300 à $700 \mathrm{~m}$ d'altitude) profondément dèchiqueté par les morsures de l'Agout et du Dadou. Ie plateau de Montredon compte 3000 habitants dispersés dans des fermes et dans de minuscules hameaux auxquels s'ajoutent les 700 habitants groupés au bourg de Labessonnié (1).

Plus de $80 \%$ de cette population vit de l'agriculture. Les ressources agricoles $y$ sont pourtant assez médiocres : une polyculture trop) diversifié pour être rentable (2) se combine, non sans mal, avec un elevage ovin laitier (Roquefort) et un élevage bovin pour la boucherie. Depuis 1851 , la population a diminué de $55 \%$. La densité agricole acluelle est inférieure à 17 et l'exode continue à un rythme qui s'accélère. Le moment semble proche où le maintien de l'équipement administratif et social ne pourra plus être normalement assuré (3). Cette érolution ne présente en elle-même rien de bien original, n'étant qu'un aspect particulier de la grande crise de dépeuplement rural.

On estime généralement que l'implantation industrielle est susceptible sinon de renverser la tendance, tout au moins de freiner la décadence. Une telle expérience est en cours dans le Montredonnais. Ces dernières années se sont ouverts plusieurs chantiers miniers dont

(1) Le Montredonnais correspond approximativement au canton de Montredon qui est formé des quatre conmunes de Rayssac, Moniroc, Arifat et MontredonIabessionnié. I.es chifres de population sont ceux du recensement de 1954 .

Jexprine toute ma gratitude à M. S. Regy, maire de Montredon, Conseiller général, à qui cetle étude doit beaucoup. Elle a été présentée au Congrès de la Fédération des Socictés Savantes I,anguedoc-Pyrénées-Gascogne de Rodez (juin 1958). En plus d'une mise à jour, on a procédé à quelques modifications de détail. Il n'y h pas d'ouvrages d'ensemble sur la région; les principaux articles utilisćs sont signalès dans les notes infrapaginales. Le lecteur pourra consulter la carte au 1/50 000 (feulles Castres et Réalmont).

(2) Les productions aquitaines telles que le blé, le maïs et la vigne se mêlent aux cultures propres à la zone montagneuse : pommes de terre, cultures fourragères (choux et navets), seigle et un peu de sarrasin.

(3) Par exemple pour entretenir les chemins vicinaux : il n'y a que 14 habitants pour $1 \mathrm{~km}$ de chemin! 
le rôle économique et social ne fait que croître. Cette implantation industriclle, d'un type tout à fait particulier il est vrai, apparaît-elle comme un érément de progrès? S'il est encore trop tôt pour dresser un bilan définitif, du moins peut-on déceler certaines tendances profondes.

Les gîtes métallifères se caractérisent dans le Montredonnais par Jcur diversité (4). Certains gisements sont liés à une intense minéralisation de contact en bordure du batholite de granito-diorite de Montredon, mais la plupart doivent être mis en relation avec le rôle de charnière tectonique qu'a joué le plateau de Montredon en bordure du Massif Central. Le long des craquelures du socle se sont injectées des solutions minéralisantes qui ont donné naissance à des filons truffés de minerais. Enrobés dans une gangue quartzeuse, l'hématite brune, la baryte, le spath-fluor et la galène se mêlent à la chalcopyrite, au manganèse, à la blende et au wolfram. Les gisements massifs sont exccptionnels; on découvre un grand nombre de petits gîtes à très forte teneur ( $85 \%$ pour la fluorine, 65 à $75 \%$ pour le fer) localisés soit à proximité immédiate de la surface (phénomène du « chapeau de fer» des gros filons de quartz) soit même en affleurement sur les versants des gorges.

Ces minerais très accessibles et facilement utilisables sont à l'origine d'une très vieille industrie minière.

I. La tradition minière (fig. 1). -. De nombreux vestiges des époques gauloise et gallo-romaine ont été découverts dans des galeries de mine profondes et bien taillées (5). On a trouvé des pics, des pelles et des lampes de mineur mêlés à des débris de poterie gauloise, de céramique romaine, de monnaies variées et même un atelier de fondeur qui remonterait peut-être à une période plus ancienne. 'Tout atteste l'èpanouissement d'une vigoureuse colonisation minière, première iorme tangible de l'occupation humaine du Montredonnais. La mise en valeur minière est, en effet, bien antérieure aux grands défrichements agricoles. Selon toute vraisemblance, l'extraction ne portait que sur des minerais précieux ou semi-précieux tels l'argent et le cuivre. La mine d'argent de Peyrebrune, située dans les gorges du Dadou, paraît jouer un rôle très important dès l'époque celtique; elle élait encore en pleine activité aux $\mathbf{I v}^{\mathrm{e}}$ et $\mathrm{v}^{\mathrm{e}}$ siècles.

Au Moyen Age, l'intérêt se concentre sur le fer, dont l'exploitation semble progresser avec rapidité à partir de la fin du xirr" siècle. Les débits soutenus de l'Agouẗ et du Dadou ainsi que les belles forêts de chênes favorisent le développement d'une très active métallurgie de type artisanal. La surface du plateau est toute taraudée de carrières

(1) Principales études minéralogiques : Gaxgloff, Etude minéralogique et métalJogénique de l'Albigeois cristallin. Buli. Soc. Hist. nat. Toulouse. t. 83, 1948; JaGRave et Gorfinet, Etudes géologiques du gisement de wolfram de MontredonLabessonnie. Ann. géol. de Belgique, Liege, t. 78, 1955, p. 44 .

(i) Caravex-Canin, Tournée géologique, archéologique... dans le département du iarn, 1869, 32 p.; In., le Tarn et ses tombeaux, 1873. 
(:) de galeries embryonnaires, ne descendant qu'exce.tionnellemeat au dessous du niveau phréatique. Ie minerai est très grossierement fondu selon la méthode catalane (la région est parsemée de no:nbreuses haldes pouvant contenir jusqu'à 70 \% de mincrai). Le fer est ainsi élaboré dans une dizaine de mollines férales qui s'échelonnent le long des gorges du Daclou, accrochant leurs barrages de poutres et de moellons aux nombreuses rugosités du fond du lit fluvial (barres de quartz ou de schistes silicifiés). Ces martinets fournissent le Montredonnais en instruments aratoires : reilles (sces d'araire), bigiossales (houes), ayssades (bêches) et bandes de roues (6). Cette aclivité métallurgique est très etroitement liée à l'agriculture; cost au début du xur" siècle que se déclenche un intense mouvement de défrichement et de colonisation.

Jusqu'a la fin du xvu" siecle, les mines sont exploitées et le's moulins tournent. ('est une intéressante ressource d'appoint, qu constitue une occupation saisonnière mettant à profit les demiloisirs que ménage le systeme de culture biennal à jachère (7). Mais, au début du xvir siècle, les bois surexploités s'épuisent et les mollines s'éteignent une à une. I'industrie minière tombe en sommeil clurant plus d'un siècle et demi pour réapparaitre ensuite complètement mètamorphosée.

De 1870 à 1900 , on note plusieurs tentatives, en général infructueuses, pour ranimer et industrialiser lextraction du fer (8). Plusieurs permis de recherches sont accordés, dont un en 1897, à la Sociéte des Hauts-Fourneaux et des Aciéries du Saut-du-Tarn (SaintJuéry, près d'Albi) qui possédait déjà des mines de fer à AlbanLe Fraysse dans le Ségala albigeois. Ies exploitations sont éphémères : après des travaux préliminaires et quelques tentatives d'extraction, c'est l'abandon plus ou moins détinitif. Vers 1890. la Sociétié du HautFourneau de Chasse (Isère) attaque le filon hématiteux du Moul'n-duRoi, dans un vallon alluent de l'Agout, a $8 \mathrm{~km}$ environ en amont de Roquecourbe. L'extraction du fer se maintient jusqu'en 1920; actuellement le chanticr est fermé mais la concession n'est pas abanclonnée.

Ces échecs répétés dérivent de causes semblables. Le's sîtes sont de pelite taille, dispersés dans des gorges difficilement accessibles et mal reliées aux routes du plateau qui forment elles-mêmes un réseau lâche et peu cohérent. Mais le principal obstacle est l'absence de voice ferrée. Le chemin de fer à voie étroite de Castres à Vabre et Murat emprunte la rive gauche de la gorge de l'Agout; il est done totalenent étranger au plateau. L'exploitation d'un minerai poncléreux d'aussi faible valeur marchancle que le fer ne paraît plus rentable de nos jours. La seule mine qui ait survécu à cette période de hatonuements

(6) L. Bantine, I.es moulines ferrières du Dadou... Bull. Soc. Sc. Arls et BellesLettres du Tarn, 1923, no 9-10, pp. 246-250.

(7) D. FAlvilik, Gengraphie agraire. Paris, Yibr. de Médicis, 1949.

(8) Pour toute cette période : Archives modernes du Tarn, Série S6M (passim). 


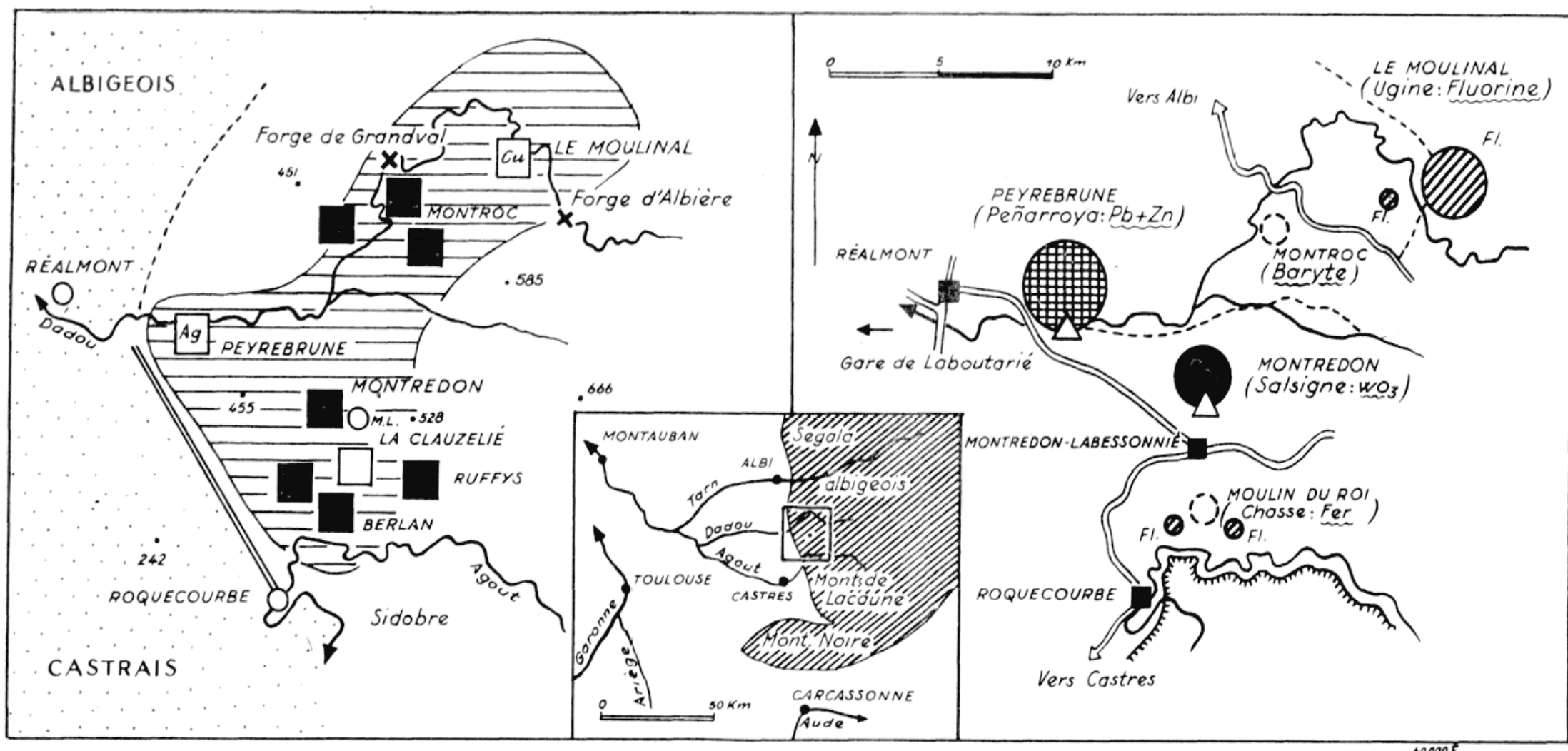

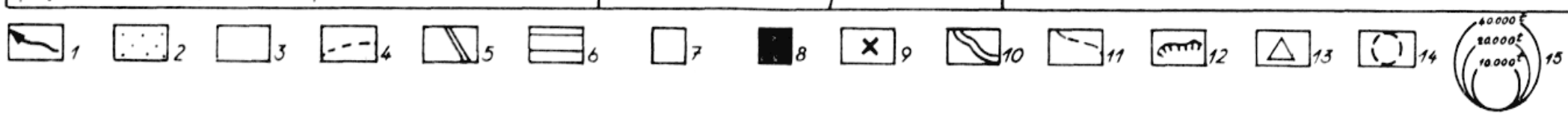

FIG. 1

\section{Les mines du Montredonnais.}

A gauche, anciennes mines; $\dot{a}$ droite, situation présente.

1. Cours d'cau. - 2. Molasse tertiaire. - - 3. Schistes primaires. - 4. Limite du massif ancien. - 5. Flexure marginale. - 6. Zone charnière fortement minéralisée. - 7. Mines gauloises et gallo-romaines (Ag : argent; Cu : cuivre). - 8. Mines de fer (xIII-Xvir siècles). - 9. Moulins à fer. - 10. Grandes routes. - 11. Mauvaises routes. - 12. Chemin de fer de Castres à Vabre et Murat. - 13. Usine de flottation. - 14. Nines concédées mais non exploitées. - 15. Extraction du « tout-venant» (en tonnes). 
est celle de Peyrebrune où l'on extrait des minerais plus chers, de plomb et de zinc. La concession date de 1832 , la mise en valeur n'a réellement débuté qu'en 1886 et se continue de nos jours.

2. Le réveil minier actuel. - Depuis quelques années, on assiste à un renouveau de l'industrie extractive qui bouleverse les conditions de l'exploitation traditionnelle (9). Ce brusque réveil dérive en premier lieu de la découverte de minerais d'alliage, produits-clés de la grande métallurgie moderne : tungstène, fluorine, manganèse et baryte. En second lieu, la présence de ces minerais rares a attiré de puissants trusts industriels et miniers qui s'en disputent, souvent âprement, l'exploitation. Mises à part les anciennes mines en sommeil, le Montredonnais est partagé entre trois grandes concessions en pleine activité : la mine le plomb et de zinc de Peyrebrune achetée par la Société Minière de Peñarroya, l'exploitation de spath-fluor au Moulinal par la Société d'Electro-chimie et d'Electro-mélallurgie d'Ugine et enfin la mine de wolfram de Montredon-Labessonnié, concédée à la Sociéie des Mines de Salsigne. Sous la vigourcuse impulsion de ces puissantes sociétés, l'extraction minière prend une ampleur de jour en jour plus grande (fig. 2 ).

a. La mine de plomb et de zinc de Peyrebrune (10), qui était gérée depuis 1893 par un groupe financier albigeois, fut absorbée en 1955 par l'eñarroya. Elle est située sur la bordure orientale du bassin permien de Réalmont, dans une zone faillée et percée de venues intrusives d'origine magmatique. I'extraction du minerai « tout venant», qui s'élevait à 13000 tonnes en 1898 et à 20000 t en 1951, est en augmentation constante : 1955, $31130 \mathrm{t} ; 1956,39396 \mathrm{t} ; 1957,41863 \mathrm{t}$. Cependant, la teneur du « tout venant » baisse de façon inquétante. Elle est passée, pour le plomb, de $3,7 \%$ en 1955 à $2,9 \%$ en 1957. Pour pallier cet effondrement, on a amélioré les procédés d'abattage et le rendement par homme-poste a doublé depuis 1949 .

Le minerai brut est enrichi sur place dans une usine de flottation, d'une capacité de $5,38 \mathrm{t} / \mathrm{h}$. Flle est équipée de tables vibratoires pour le traitement de la galène et de trieuses électro-magnétiques pour l'enrichissement des blendes. En 1957, la production de concentrés s'établissait ainsi : galène, $1464 \mathrm{t}$ à 77 \% de plomb; blende : $1434 \mathrm{t}$ à $54,8 \%$ de zinc. Les concentrés sont transportés par camions à la petite gare de Iaboutarié, distante de $14 \mathrm{~km}$; de là, ils sont expédiés aux foncleries de Peñarroya à Noyelles-Godault, près de Calais.

Pevrebrune occune une nlace infime sur le marché français: environ $5 \%$ de la production de plomb et seulement $0,2 \%$ de celle de zinc. Ia mine. cxnloitéc dès l'énoque gauloise et d'une façon souvent peu méthodique, est en voie d'épuisement. A la fin de 1957, les

(9) Pour les statistiques : Rapport du Préfet au Conseil Général. Albl, publ. annuelle, 1951 à 1957 .

(10) P. Fénelon, les mines françaises de plomb et de zinc. Rev. Géagr. Pyrénés, 195., pp. $272-291$. 
réserves étaient estimées seulement à $3840 \mathrm{t}$ « à vue $\gg, 9700 \mathrm{t}$ 《probables» et $90000 \mathrm{t}$ 《possibles ». Une importante campagne de prospection est en cours, mais l'avenir de Peyrebrune réside moins dans sa propre richesse que dans celle de Peñarroya.

b. Ia inine de spath-fluor du Moulinal (11), près de Saint-Jean-deJanes, qui a été concédéc en 1943 à Ugine, paraît avoir un avenir meux assuré. L'exploitation porte sur un puissant filon quartzeux parcouru par des veines de s!ath-fluor. Ia production de la mine,

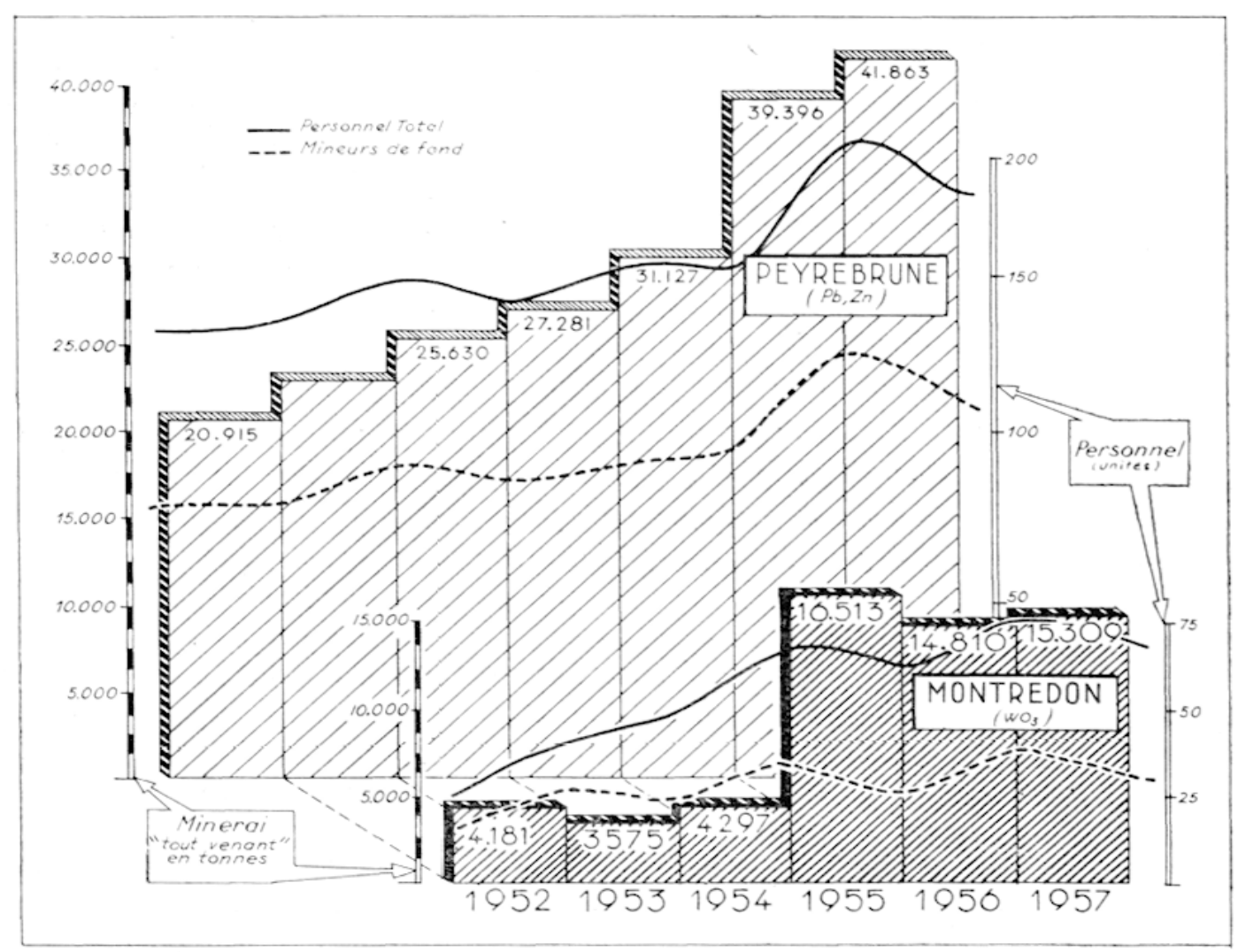

Fig. 2

L'évolution de la production et des effectifs dans les mines du Montredonnais.

ajoutéc à celle de se's annexes dispersées sur le plateau, dépasse 20000 t. Le minerai en place est presque pur : il contient couramment $85 \%$ de fluorine. Aussi les quatre cinquièmes sont-ils vendus directement, le reste est expédié à la grande laverie gravimétrique d'Ligine à Pierre-Bénite (Ihhône) pour être enrichi. C'est une mine en

(11) I.e spath-fluor sert à fabriquer la cryolite artificielle qui est un « fondant 》 utilisé dans l'industric de l'oluminium et des aciers spéciaux. On lemploie aussi sous forme dacide fluorhydrique et de sels derives dans les industries chimiques et pharmaceutiques. 
pleine expansion qui doit fournir de 15 a $20 \%$ de la production nationale.

c. La mine de wolfran (12) à $2 \mathrm{~km}$ du bourg de MontredonLabessonnié fut obtenue de haute lutte en 1953 par la Société des Mines de Salsigne. Il s'agit d'une très riche minéralisation au contact des cornéennes et de la coupole granitique. La production, qui ne portait en 1354, que sur 4297 t de minerai tout-venant, s'élève à $16500 \mathrm{t}$ en 1956; mais elle n'arrive plus a se maintenir à ce niveau $(15309$ t en 1957). Ia teneur du minerai brut a baissé de $1,27 \%$ (1955) à $0,9 \%$ (1957). Une laverie par flottation installée pas loin de la mine, permet de traiter à plein rendement $7 \mathrm{t}$ de tout-venant à l'heure. Ia production des concentrés se maintient avec difficulté :

1955 : 104 t de concentrés à $68,6 \% W^{3}$, soit 71 t de minerai pur

$1956: 130 \mathrm{t} \quad \cdots \quad 68,5 \% \quad \ldots-85 \mathrm{t}$

$1957: 64$ t $\quad \ldots-66,3 \% \quad \ldots \quad 42,5$ t $\quad$ -

A ces dificultés techniques s'ajoutent de sérieuses perturbations financières dues à l'effondrement du cours du wolfram sur le marché international. L'exploitation est en grande partie maintenue artificiellement. 'Toutefois, les nouvelles perspectives d'utilisation du wolfram (fusées) et les appréciables réserves de la mine $(40000 \mathrm{t}$ à vue et plus de 200000 t probables) paraissent assez favorables. En 1957. cette mine fournissait $13 \%$ de la production française de wolfram.

Les mines montredonnaises occupent sur le marché national une place qui n'est pas à dédaigner. Elles représentent une indiscutable réussite technique el financière. Mais leur véritable intérêt se situe sur le plan strictement local, en fonction du milieu rural.

3. La mine et la crise rurale. -... L'ancienne métallurgic artisanale du fer faisait partie intégrante de l'économie agricole. Au contraire, l'industrie minière actuelle est surimposée au milieu rural. Par ses prolongements humains et économiques, cette implantation pose deux sérieux problemes : celui du mineur-paysan et celui des rapports de la mine avec l'économie agricole.

a. Une solution provisoire : le mineur-paysan. -... Les effectifs, qui ètaient de 238 ouvriers en 1955, se sont élevés à 300 en 1956 pour retomber à 280 en 1957 (fig. 2) : Peyrebrune 169 ouvriers; Montredon 70; Moulinal 30. Une dizaine d'ouvriers sont, en outre, éparpillés dans divers chantiers. Ia moitis à peine se recrutent dans les quatre communes du Montredonnais, ce qui représente, il est vrai, de 6 à $7 \%$ de leur population active. Mis à part une dizaine d'Espagnols et de Portugais, ce sont de jeunes agriculteurs qui conservent presque tou:ours des attaches étroites avec la terre.

(12) Le wolfram (minerai de tungstène) sert à la fabrication de ferro-alliages, des flaments de lampes électriques, des tallants de fleurets de marteauxperforateurs, etc. 
Les mineurs-paysans ne constituent pas une catégoric socialement homogène et encore moins une classe. Ils ont conservé leur place dans la hiérarchie paysanne et c'est là, certainement, le meilleur critère de leur différenciation. Le type le plus répandu est le mineurjournalier agricole qui se loue pour les gros travaux saisonniers ot constitue le reste du temps une main-d'xuvre volante. Il habite en général au bourg et cultive un jardin potager pendant ses rares loisirs. Très nombreux sont aussi les mineurs fils de petits propriétaires et de métayers qui aident quotidiennement leur famille. Enfin, il $y$ a le cas des mineurs-chefs d'exploitation agricole qui doivent mener de front deux occupations épuisantes.

En voici deux exemples (13) : M. est un manouve de galerie; il possède à $11 \mathrm{~km}$ de son lieu de travail, où il se rend à motocyclette. une maison et un petit « bien de village » de 4,9 hectares qu'il cultive pendant ses journées de repos; il y récolte des pommes de terre pour l'année, divers légumes et du blé qu'il échange contre une partie de son pain. Le cas de N., mineur de fond, est très différent; il est propriétaire, à $5 \mathrm{~km}$ de la mine où il travaille, d'une exploitation de 13,8 hectares : 2,1 ha de céréales dont 1 ha de blé; 0,4 ha de vigne. 2 ha de prairies artificielles, 4 ha de prés naturels, ce qui lui permet d'élever 4 vaches et 2 pores. Malgré l'aide de deux membres de sa famille, il ne peut cultiver sa terre qu'en rognant sur son temps de sommeil. De telles combinaisons sont-elles souhaitables?

Si on se place au point de vue de l'individu, les apparences ne sont pas tellement défavorables. Le mineur-paysan ne se plaint d'ailleurs pas trop de son sort. Il bénéficie d'avantages inconnus du paysan pur : salaire garanti et horaire fixe. semaine de 40 ou 48 heures selon les mines, 15 jours de congés payés coïncidant généralement avec l'époque de la moisson, afliliation à la sécurité sociale, à la Société de Secours Minicr etc, avantages qu'il cumule avec le revenu de la terre.

Mais les dangers d'un tel système sont évidents. Ia mine et l'agriculture ne sont pas des activités complémentaires, et demandent toutes deux de grosses dépenses d'énergie. Ice mineur-paysan voit loubler sa peine, mais non son profit. D'une part, son instructio: générale est très sommaire (certificat d'études tout au plus) et ses connaissances professionnelles sont nulles, ce qui le condamne à rester un simple manouvre. D'autre part, à cause du manque de temps et de la fatigue, il est obligatoirement amené à négliger son exploitation qui ne lui fournit alors qu'un bien maigre complément. Une grande partie du travail agricole (entretien du betail, culture de plein champ) incombe à sa femme ou à ses parents dont la condition est bien plus pénible que dans le cas d'un genre de vic purement rural. Les sociétés minières, tenant très largement compte de l'appoint

(13) Source statistique : Recensement agricole de 1956, dont los fiches nous ont été aimablement communiquées par la Direction régiónale de I'I. N.S.E. E. à Toulouse. 
agricole et jouant sur le manque de sens syndical du mineur-paysan, ne distribuent que des salaires de base à peine supérieurs au S.M.I.G., ce qui a pour contre-partie de river le mineur à son champ et de fermer le cercle vicieux. L'importance accordée aux primes de rendement, souvent substantielles il est vrai, accentue la tendance déjà marquée au surmenage physique. Non légagé de ses origines paysannes, le mineur-paysan conserve une mentalité de petit possédant, qui contrasle avec la prolétarisation croissante de son genre de vie. Mloins que toute autre forme d'implantation industrielle, l'extraction minière peut se prêter à la formation d'un genre de vie mixte. Si le travail à la mine ne se ressent pas trop de ce cumul, mis à part un certain absentéisme au moment des gros travaux (fenaison, moisson), toute la vie montredonnaise subit le contre-coup de cette combinaison sociale.

b. La mine et ses incidences économiques. -- En 1957, les diverses sociétés minières semblent avoir distribué dans le Montredonnais, environ une quarantaine de millions de francs, essentiellement sous forme de salaires, soit une moyenne de $12000 \mathrm{~F}$ par habitant, ce qui n'est pas négligeable pour une région aussi pauvre.

Cet appoint de revenus a très largement contribué à ranimer le bourg de Labessonnié. Ce petit centre agricole, étouffé par la proximité de Castres (14) abrite une grande partie du personnel de la mine de de wolfram et on voit s'élever quelques bâtiments d'habitation. Ces activités nouvelles lient de plus en plus étroitement l'avenir du bourg à celui de la mine et tendent à détourner Labessonnié de sa fonction de bourg rural. Cette évolution n'est pas sans danger (15).

Ces nouvelles ressources sont trop modestes pour s'investir dans le domaine agricole : elles sont justes suffisantes pour subvenir aux besoins des mineurs-paysans et de leur famille. En ouvrant sur place un marché de main-d'oeuvre important à l'échelle du Montredonnais, les mines donnent l'illusion de participer au maintien de la population. Il est incontestable que beaucoup de jeunes paysans qui, dans l'immédiat, auraient émigré, se sont embauchés sur place. Mais, certainement, un nombre au moins aussi grand de jeunes qui seraient restés à la terre, ont succombé au mirage du salaire ouvrier. De toute manière, la mine ne peut pas fixer la population d'une façon définitive; elle n'est que la première étape d'un abandon sans espoir de retour.

En effet, le genre de vie mixte n'a qu'une existence très éphémère. Si le mineur de la première génération reste avant tout un paysan, celui de la seconde génération tend à devenir un simple jardinier. Le contraste est frappant entre la mine récente de Montredon, qui compte une majorité de mineurs-paysans, et la vieille mine de Peyre-

(14) Mme G. Bertraxi, Le rayonnement de Castres. Diplóme d'Etudes Supérieures, Institut de Gégraphie de Toulouse (en préparation).

(15) M. S. Regy, maire de Montredon, nous écrit : * La mine est la piéce maltresse du chef-lieu de canton. Sa fermeture sera l'enterrement de Montredon. \$ 
brune où beaucoup d'ouvriers sont détachés de la terre. Dans cette dernière mine, l'évolution a été poussée jusqu'à son terme : les mineurs originaires de la région cèdent la place aux Espagnols et aux Italiens qui s'adaptent mieux au rude travail du mineur. Un processus semblable menace aussi la mine de wolfram qui, déjà en 1956, comptait un peu plus de $12 \%$ d'étrangers. A ce stade, l'extraction minière ne joue plus son rôle modérateur sur l'immigration, et n'a pas été plus qu'un palliatif temporaire.

Mais le principal danger est que la mine, par sa seule présence, aggrave une crise agricole latente. Le travail de la terre par le mineurpaysan a de nombrcux inconvénients. Il aboutit à unc lente stérilisation des exploitations : resiriction de la partie cultivée aux meilleurs fonds, terres mal chaulées et mal engraissées, bétail négligé, chemins et bâtiments mal entretenus En outre, la mine détourne beaucoup de jeunes de l'idée de progrès agricole; on compte peu de tentatives pour spécialiser les exploitations et pour améliorer les rendements : ce serait pourtant assez. facile, du moins en ce qui concerne l'élevage ovin et bovin.

L'industrie minière est une ressource instable, menacée par l'épuisement des gîtes et soumise aux incertitudes du marché international. Elle n'en demeure pas moins indispensable au maintien d'un précaire équilibre économique. Mais elle a tendance à masquer les véritables problèmes, qui sont essentiellement d'ordre agricole. L'agriculture, qui tend à évoluer lentement vers un élevage demi-spécialisé, reste à coup sûr, nalgré sa médliocrité générale, la base fondàmentale de l'économic montredonnaise et presque la seule ressource d'avenir. 